m16.002

\section{Cs-Correction and Electron Holography: Exciting TEM-Methods for Solving Nanoquestions}

\author{
Michael Lehmann \\ Triebenberg Laboratory, Institute of Structure Physics, University of \\ Dresden.Michael.Lehmann@Triebenberg.de

\section{Keywords: HRTEM, electron holography, electron optics}

The macroscopic properties of modern materials and nanostructured systems are determined by their atomic structure. Therefore, for a tailored design of materials with optimized properties, the structure-property relationships have to be investigated making a structural characterization in terms of the nanoquestions "which atoms are where, which fields are around" indispensable. These nanoquestions are tackled by well-elaborated characterization methods such as e.g. X-ray and neutron diffraction, SXM and AFM, and high-resolution transmission electron microscopy (HRTEM). However, every method has its own pros and cons: Despite the fact that diffraction methods are able to precisely determine lattice parameters, their spatial resolution is limited due to averaging over the illuminated area with a diameter of about $1 \mu \mathrm{m}$. Thus, specific structure modifications of real-world samples on the nanometer scale such as e.g. interfaces, grain boundaries, inclusions, and defects cannot be resolved locally. SXM and AFM are unrivalled in investigating surface structures up to atomic resolution; however, they have no access to the interior structure of the sample. HRTEM is the method of choice offering the unique possibility of spatially resolved characterization of the interior structure of real-world structures with atomic resolution. In the past, HRTEM was hampered by the spherical aberration (Cs) of the objective lens causing a blurring hence an averaging over $1 . .2 \mathrm{~nm}$ in the image plane. Since the late 90's, hardware Cs-correctors [1,2] are available allowing the correction of aberrations up the 3rd order, which marks a giant step in HRTEM. Cs-correction reduces the blurring dramatically so that the signal piles up at atom positions well above noise. Despite this huge progress, the phase contrast problem of imaging still persists. Therefore, Cs-correction is combined with off-axis electron holography [3], where an interference pattern ("electron hologram") is recorded in the TEM. By numerical reconstruction, the complete information of the electron wave is available quantitatively in amplitude and phase as well as in real space and in Fourier space. Additional beneficial capabilities are almost perfect zero-loss energy-filtering with about $10^{-15} \mathrm{eV}$ as well as linear imaging. All this allows a comprehensive processing and analysis of the recorded data: Residual aberrations also of higher orders, which cannot be completely avoided, can be corrected by an a-posteriori fine-tuning. Diffraction patterns are determined from areas of the size of a few unit cells allowing a local investigation of crystal orientation. Imaging in the light of single reflections enables the sophisticated analysis of diffracted waves. Moreover, large area phase-shifting structures like electric/magnetic domains and biological/polymer specimen can directly be investigated by electron holography. Therefore, modern TEM-methods cover a wide range of applications [4] from dopant-profiling in semiconductors and field measurements of small magnetic clusters up to holographic materials analysis with single atom sensitivity.

[1] Rose H., Optik, 1990, 85, 19

[2] Haider M., Uhlemann S., Schwan E., Nature 1998, 392, 768.

[3] Lichte H., Ultramicroscopy 1986, 20, 293.

[4] Lichte H., Lehmann M., Adv. Imag. Electr. Phys., 2002, 123, 225. m16.003

\section{Organic and inorganic assemblies}

\section{Nina L. Smirnova}

Department of Geology, Moscow State University, Moscow, Russia. E-mail: snl194@mail.ru

\section{Keywords: organic polycycles, planar assemblies, design}

A minal is a term authored by Gustav Tschermak (1836-1927). A minal is a real or theoretical end member of an isomorphic series of chemical compounds of constant composition. The structural minal (sminal) is a real or theoretical end member of polytypic, heteropolytypic series of structures (structure types). Some of them exist in nature; some are virtual, existing not overtly but as thought constructs. All structures are constructs of sminals. Every sminal consists of skeleton invariant and variant part. Sminals exist as latent constructs, then as defects and at last as ordered defects - new superstructures. Some examples of these phenomena are considered. All structures consist of 11 Kepler-Shubnikov (Archimedean) nets. They contain latent binary assembles (AS) of identical n-gons 33 (sheared edge), 3v3 (sheared vertex), 44, 4v4, 66, 6v6, 88,.12.12 (.12 - digit number), of different n-gons $34,3 \mathrm{v} 4,36,3 \mathrm{v} 6,3.12$, $46,48,4.12,6.12$. Some extra binary AS were revealed among 21 types of vertices and 4-uniform tiling 45678: 37, 38, 39, 3.10, $3 \mathrm{v} 12,3.15,3.18,3.24,3.42,45,4 \mathrm{v} 6,47,4 \mathrm{v} .12$, 4.20, 55, 56, 57, $58,5.10,5.20,67,68,7.42,8.24,9.18, .10 .15$. AS consisting of 3 $\mathrm{n}$-gons are denoted as ratios 3 (identical n-gons), 1/2 (two different n-gons), 1/1/1 (three different n-gons). AS consisting of $4 \mathrm{n}$-gons are denoted as $4,1 / 3,2 / 2,1 / 1 / 2,1 / 1 / 1 / 1$. Realized linear AS were revealed: 3 - a, 34 - ab, 35 - ab, avb, 36 - avb, abb, 4 - a, ava, 45 - ab, 46 - ab, avb, 48 - ab, 5 - a, aa, aaa, aavaa, 56 - ab, avb, aab, avab, aba, abb, bab, bavb, abbb, babb, bbbva, bavab, aabbb, avabbb, abbba, aabba, abbab, bbbab, abbbba, abbbbba, babbbbbab, 57 - ab, 58 - ab, 6 - a, aa, aaa, aava, avava, aaaa, aavaa, aaaaa, aаaаa, aаaаaаa, aaavaaaa, aаaаaаaа, 67 - ab, aab, aba, bab, abb, 68 - ab, aba, 7 - a, 8 - a, 356 - abbc, 576 - abc, 658 - abc, 6745 - abcd, abcdcdcba. Realized central AS were revealed: $6,46,56,58,67,69,456,567,568$. Realized ratios of n-gons were revealed for linear and central (c) AS: 1, $2,3,4,5,6,7,8,9,1 / 1,1 / 2,1 / 3,1 / 4,1 / 5,2 / 2,2 / 3,2 / 4,2 / 5,2 / 7$, $2 / 8,2 / 9,2 / 10,3 / 4,3 / 5,4 / 4,4 / 8,1 / 1 / 1,1 / 1 / 2,1 / 1 / 3,2 / 2 / 8,4 / 4 / 4$, $1 / 1 / 1 / 1,2 / 2 / 2 / 3$. Vertices in AS are shared by $1,2,3,4$ different n-gons $(1,2,3,4$ different valence, DV). The set of AS was split into 4 subsets: 1) DV 1, 2) DV 21, 3) DV 213, 4) DV 2134. Linear, Planar Complication Scheme (PCS) of vertex quantity ratios were composed. Basic ratios in quotas are latent: "1/1", 16/16, 6/7, 5/6, "4/5", c12/15, 12/15/2, 16/20, 16/20/4/1, "3/4", c6/8, 6/8/2, 9/12/5/1, 12/16, 8/11, 8/11/4/1, 16/22/0/1, "5/7", 10/14, 17/24/0/2, 2/3, c6/9, 691, 8/12, c8/12, 8/12/2, 8/12/4, 8/12/4/1, 17/26/0/2, 9/14/1, 7/11/1, "5/8", 10/16, c10/16, 10/16/4, 8/13, 8/13/2, 8/13/4, "3/5", 6/10, 12/20, 7/12/1, 4/7, 8/14, 8/14/4, 8/14/6, 5/9/1, 11/20, 6/11, 6/11/1, 6/11/2, "1/2", 2/4, 4/8, 5/10, 5/10/1, 6/12, c6/12, 6/12/2, 6/12/4, 6/12/6, 7/14, c6/13, 6/13/1, 5/11/1, 5/11/2, 5/11/4/1, 4/9, 4/9/2, 3/7/1, 5/12, 5/12/2,"2/5", 4/10, 4/10/2, c6/15, 5/13, 3/8/1, 4/11, "1/3", 2/6, 3/9/1, 4/12, 4/12/1, 4/12/0/1, 3/10, 2/7, 3/11, "1/4", 2/8, 2/9, "1/5", 2/10, 1/6, "1/7", 2/14, 1/8, 1/9, 1/10. Ratios were put into Weiss-Brococci-GoldschmidtFarey-Smirnova PCS.

[1] Grunbaum B., Shephard G. C. Tilings and patterns W.H. Freeman and Co, New York 1987. 